\title{
A COMPARATIVE STUDY OF OPEN AND LAPAROSCOPIC CHOLECYSTECTOMY
}

Shekappa C. Malagimani ${ }^{1}$, Somashekhar Samagandi², Ishwar Hosamani'3 ${ }^{3}$ Yashwanth C. T4, Vasanta S. $\mathrm{C}^{5}$

\section{HOW TO CITE THIS ARTICLE:}

Shekappa C. Malagimani, Somashekhar Samagandi, Ishwar Hosamani, Yashwanth C. T, Vasanta S. C. "A Comparative Study of Open and Laparoscopic Cholecystectomy". Journal of Evolution of Medical and Dental Sciences 2014; Vol. 3, Issue 08, February 24; Page: 2016-2023, DOI: 10.14260/jemds/2014/2097

INTRODUCTION: Gallstones disease is one of the commonest general surgical problems requiring operative intervention in India. 10-15\% of adults in the developed countries will develop gallstones. In the United States more than 500,000 cholecystectomies are performed per year, making it the commonest upper abdominal surgery performed. Ever since Langenbuch performed the first cholecystectomy more than a 110 years ago. Phillipe Mouret performed the first laparoscopic cholecystectomy in 1987, the surgical world has been caught in a storm. This surgery has been popularized by both the media and the surgical community as being virtually pain free and cosmetically superior to all the other approaches to gallbladder removal. Laparoscopic cholecystectomy has rapidly expanded worldwide over a very short span of time. Therefore, the aim of our study was to compare prospectively the outcome, in terms of morbidity and mortality, in laparoscopic versus open surgery.

KEYWORDS: laparoscopic and open cholecystectomy.

\section{AIMS AND OBJECTIVES:}

A. To find out the indications and contraindications for laparoscopic and open cholecystectomy.

B. To compare the morbidity and mortality involved in laparoscopic and open cholecystectomy.

C. To compare the operative time of each.

D. To find out the conversion rate in laparoscopic group.

REVIEW OF LITERATURE: The current therapy of gallstone disease is based on thorough understanding of the anatomy, physiology and biochemistry responsible for the pathogenesis of gallstones. Perhaps no other disease can boast of having such an extensive armamentarium of treatment modalities lined up against it. ${ }^{1}$ Ever since the first cholecystectomy in $1882^{2}$ the treatment of gallstone disease has undergone a considerable change. The general tendency has been towards the procedures which are less likely to be associated with the ill effects of surgery mainly the creation of a surgical wound and its attendant complications and the small but significant risk of exposure to general anesthesia. At various times the gastroenterologists and the interventional radiologists ${ }^{3}$ have staked claim for a superior and less traumatic therapy for what is essential a surgical disease for open cholecystectomy. Open cholecystectomy is a surgery in which the gall bladder is removed should be considered. The gold standard ${ }^{4}$ against which all other approaches should be judged. Open cholecystectomy has been performed for over 110 years now via various incisions ${ }^{5}$ with an impeccable tract record of safety and minimal morbidity and mortality.6. The only contraindication has been unacceptably high risk of general anesthesia. ${ }^{7}$ Indeed Langenbuch was prophetic when he said, the gallbladder should not be removed because it contains stones but because it form them. The 
main disadvantage that has come to be associated with open cholecystectomy is the creation of a surgical wound and its sequel. The scar which is difficult to conceal, the incision pain which in some cases may last of more than 2 years. ${ }^{8}$ The requirement for a long post-operative hospital stay and subsequent delay in return to normal activity 9 . The loss of income suffered for the same is another important factor. It should therefore come as no surprise that so many alternative therapies are still being practiced.

Laparoscopic cholecystectomy first performed by Mouret in 1987.10 This procedure has since revolutionized the treatment of gallstones disease. The large surgical wound required for access has been virtually eliminated. There has been a sharp reduction in postoperative hospital stay and convalescence. A quicker return to normal productive life has been seen.

Initially greeted with great skepticism it has now been declared the procedure of choice for symptomatic gallstone disease. ${ }^{11}$ Although laparoscopic cholecystectomy has nearly replaced open cholecystectomy, surgeons still perform a small but significant number of planned open cholecystectomies. ${ }^{12}$

MATERIALS AND METHODS: It is a prospective study designed in which all the patient admitted in surgical wards of the hospital for the study period of April 2011 to March 2012 are source of data. They were subjected for thorough history, physical examination and available investigations required for study and particular note was made on

1. Presence or absence of common bile duct stones, documented by history, radiological and biochemical investigations.

2. Fitness for surgery, particularly with respect to cardiopulmonary status.

Laparoscopic cholecystectomy: All the patients are operated in the supine position. We routinely introduce a nasogastric tube to decompress the stomach. All patients were catheterized before being shifted to the operation theatre. After skin preparation and draping, the head end of the table is lowered, a one cm skin incision is made in the supraumblical skin fold, from which the Veress needle is introduced into the peritoneal cavity, directed towards the sacral hollow. The position of the needle in the peritoneal cavity is confirmed by the free movement of the needle, the saline drop test and pressure recordings taken by the carbon dioxide insufflators.

Pneumopertioneum is created by an electronic CO2 insufflators set at $12 \mathrm{~mm}$ of $\mathrm{Hg}$ with a flow rate of 1 liter per minute. Once the intra-abdominal pressure reaches the preset level, the head end of the table is now raised and the table tilted to the left side to displace bowel away from the right upper quadrant. The Veress needle is replaced by the $11 \mathrm{~mm}$ trocar and cannula.

The pressure now being maintained by continuous insufflations through this cannula after removal of the trocar. The insufflations is continued to maintain a constant intra-abdominal pressure of $12-14 \mathrm{~mm}$ of mercury. Then the telescope is inserted through the umbilical port and the remaining cannulas are introduced under direct vision. We use $10 \mathrm{~mm}$ port to the right of midline below the costal margin and two $5 \mathrm{~mm}$ port in the anterior axillary line at the line at the level of umbilicus.

The fundus of the gallbladder is identified and held by a pair of grasping forces being introduced via the $5 \mathrm{~mm}$ anterior axillary port and the other grasping forceps being applied at the neck. Traction being applied cranially at the gall bladder fundus by the grasping forceps holding the fundus and infero laterally at infundibulum by the other forceps going through the other $5 \mathrm{~mm}$ port. 
Any adhesions between the gallbladder and the surrounding structures (stomach, duodenum, colon, and omentum) are separated by blunt dissection using dissection forceps introduced through the upper $11 \mathrm{~mm}$ port. Following this the cystic duct and artery are dissected out. The cystic artery is divided in between clips. Clips are applied, two on the common bile duct side of the cystic duct and one on the gallbladder side.

The cystic duct is divided in between. The gall bladder is then dissected off the gallbladder fossa using the hook, spatula and dissecting forceps. Hemostasis is secured using electrocautery. The dissected gallbladder is placed above the right lobe of the liver with the grasping forceps holding it at its neck. A drain is introduced through the $5 \mathrm{~mm}$ port in the anterior axillary line and placed in the gallbladder fossa where ever needed. The gall bladder is held by grasper to be taken of the $10 \mathrm{~mm}$ umbilical port. Stones and bile are removed if they cause obstruction. The umbilical incision is closed in two layers, inner fascia with 1-0 vicryl or 1-0 prolene and skin with 2-0 vicryl, the other skin incisions are approximated with 2-0 silk sutures only.

Open cholecystectomy: The patient is placed in the supine position with a sandbag placed under the upper abdomen, after cleaning and draping, a $5 \mathrm{~cm}$ transverse incision is made in the right subcostal region midway between the xiphisternum and the umbilicus just short of the midline. The oblique abdominal muscles are cut with cautery. The peritoneum is opened. Dever's retractors are used to retract the liver, the colon, the stomach and the duodenum. The gall bladder is identified. Its fundus is held with a Kelly's clamp, another Kelly's is used to hold the gallbladder at the Hartman's pouch.

The adhesions between duodenum and gallbladder, right flexure and gall bladder are released by blunt and sharp flexure and gall bladder are released by blunt and sharp dissection. The cystic duct and artery are dissected ligated and cut. The gallbladder is dissected off its fossa. Hemostasis is achieved. The abdomen is closed in two layers inner with 1-0 vicryl and outer with 1-0 prolene. The skin is closed with 2-0 silk interrupted sutures.

OBSERVATIONS AND ANALYSIS: The results of our comparative study from April 2011 to March 2012 in which 96 patients participated are now summarized.

A total number of 40 patients were offered laparoscopic cholecystectomy, of these 13 were males and 27 females. There were 6 conversions to open surgery ( 4 males, 2 females) of the 56 patients taken up for open cholecystectomy, 23 were males and 33 females. In our study all conversions were considered as open cases. (see Fig. 1). 


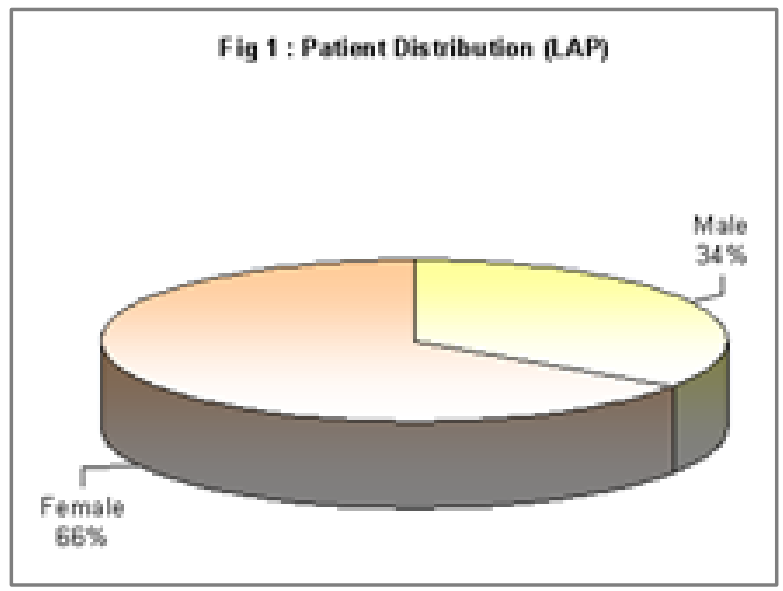

Figure 1

The average age of males undergoing laparoscopic cholecystectomy was 48.92yrs (range 2370yrs,) and for those who underwent open surgery, the average age was 41.52yrs (range 22-70yrs, $\mathrm{P}$ not significant). In case of females taken up for laparoscopic cholecystectomy the average age was 44.18 yrs. (range: 23-68yrs,) and for those who underwent open surgery the average age was 47.12yrs (range: $18-75 y$ rs, $P$ not significant). 17 patients (17.7\%) above the age of $60 y$ rs were taken up for cholecystectomy. The patients $(92.7 \%, n=89)$ were most often symptomatic in the $2^{\text {nd }}$ to $6^{\text {th }}$ decade of life $(\mathrm{P}<0.001)$ for both males and female in either group. Most of our patients had grade II (n-45, 46.87\%) or Grade I (n-33: 34,3\%) symptoms. 8 patients presented with Acute cholecystitis. (Grade III symptom and 10 patients with Grade IV).(see fig. 2)

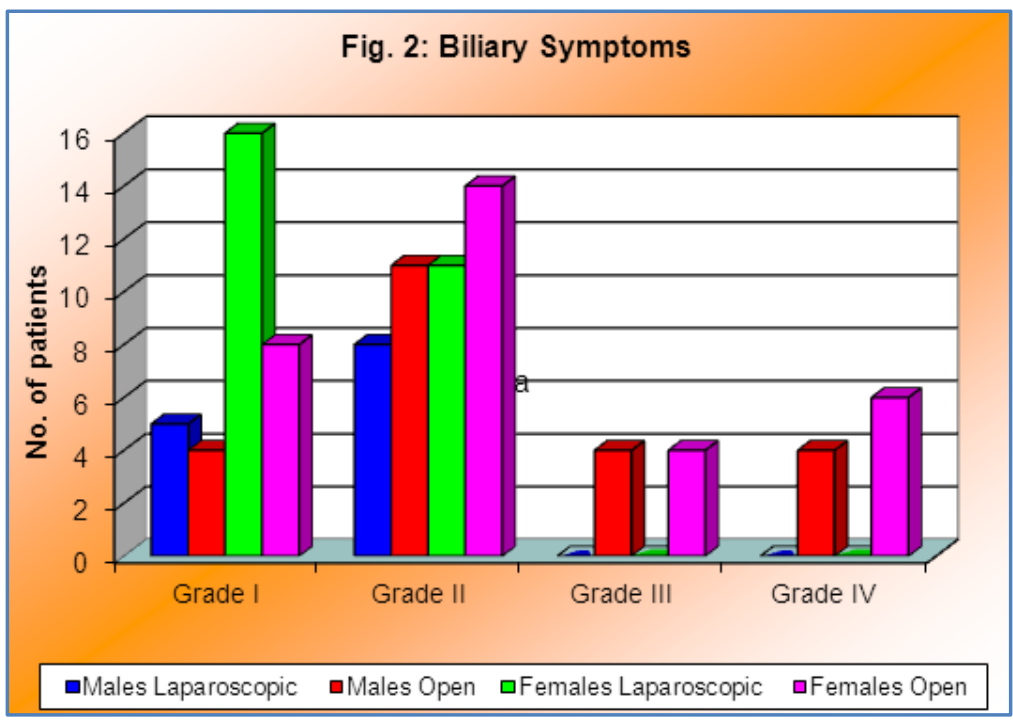

Figure 2

Operative procedure: Using the criteria defined in the methodology the mean operation time for males undergoing laparoscopic cholecystectomy was 90.82 minutes (range $-60-180 \mathrm{~mm}$ ) as compared 81.1 minutes (range $=60-210$ ) taken for open cholecystectomy ( $\mathrm{p}-$ not significant). For females undergoing laparoscopic cholecystectomy, the mean operation time was 88.12 minutes 
(range 60-120) as compared to 74.4 minutes (range 50-180) for open cholecystectomy. (p-not significant) (See fig. 3).

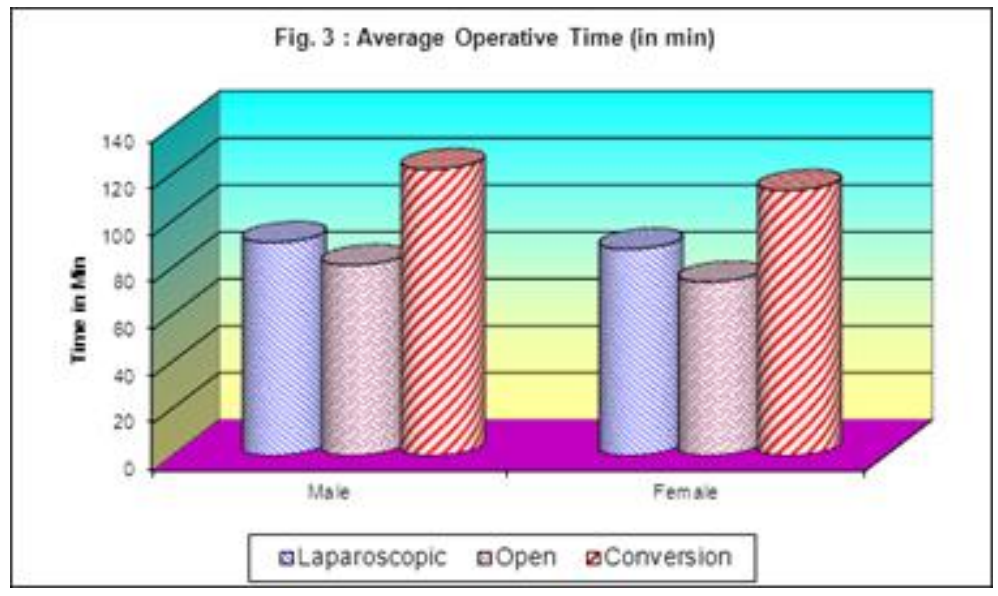

\section{Figure 3}

Of the 56 patients taken up for open cholecystectomy a right sub-costal incision was used in all 56 patients.

Of the 46 cases taken up for laparoscopic cholecystectomy 6 cases were converted to an open procedure giving a conversion rate of $13 \%$.

Morbidity: i) It is quite evident that on the day of operation $65 \%(n=26)$ of the patients undergoing laparoscopic cholecystectomy scored II and III on the visual Analogue Scale (VAS). As compared to $64.28(n=36)$ of the open group who scored VII or VIII on the same scale for pain. This clustering of cases was significant $(\mathrm{p}<0.001)$.

Postoperative stay: The average stay for patients undergoing laparoscopic cholecystectomy was 2.68 days (range 2 - 11 days males 2.56 days, females 2.81 days). Conversion to open surgery increase the post-operative stay to $6.65 \%$ days (Range 4-12 days, males 7.1days, females 6.2 days).Patients who underwent open surgery stayed for a mean duration of 4.95 days (range 3-15 days males 4.8 days, females 5.1 days) (p- not significant)

DISCUSSION: Symptomatic gall stones account for a sizeable proportion of surgeries performed in our surgical practice in India. It often affects people in their economically and socially productive age. Until recently the only surgical option available for permanent alleviation of symptoms was open cholecystectomy. But, this too was far from perfect in the sense that it led to the creation of the large surgical wound with its attendant morbidity and involved a long convalescence. The non-surgical therapies have proved ineffective by having severe restrictions on their applicability. Therefore, when laparoscopic cholecystectomy burst upon the seen it was hailed that as the panacea for patients with gall stones diseases. It cured the patients and so with minimal disability.

Of the 40 cases taken up for laparoscopic cholecystectomy 6 cases were converted to open surgery giving a conversion rate of $13 \%$. This was comparable to the conversion rate of $1.8-14 \%$ reported in world literature. Most authors would say that a 5-10\% conversion rate is very acceptable in unselected series. 
The most common reason for conversion that we experienced was a contracted or thick walled gallbladder with obliteration of the Calot's triangle. This was the sole reason for conversion in $66 \%(n=4)$. The average operation time for a laparoscopic cholecystectomy was 87.3 minutes (60180) as compared to 78.2 minutes for the open procedure. This difference was not statistically significant. The mean operation time as reported in literature varies from 39-118 minutes. On the first post-operative day most patients undergoing laparoscopic cholecystectomy experienced lesser pain as quantified by the visual analogue scale (VAS) and the verbal response score (VRS).

As far as quantification of pain goes, we had results similar to other authors with lower mean values for VAS ${ }^{14}$.The mean postoperative stay after laparoscopic surgery was 2.68 days as compared to 4.98 days for those who underwent open surgery. In conversion group it is 6.65 days. The average postoperative stay reported in literature ranged from 0.98-3.0 days for laparoscopic cholecystectomy. The post-operative stay for an open cholecystectomy ranges from 6.2-6.6 days as reported in literature.

We had wound infection rate of $5 \%(n-2)$ in laparoscopic group compared to $14.2 \%(n=8)$ in open group.

CONCLUSION: Gallbladder stone without CBD stones without acute cholecystitis were main indications for laparoscopic cholecystectomy and CBD stones, acute cholecystitis, dense adhesions were contra indications for laparoscopic cholecystectomy in our study.

Unacceptable anesthesia risk was the only contraindication for open cholecystectomy.

Laparoscopic cholecystectomy proved to be superior to open cholecystectomy as regards the postoperative morbidity. There was no significant difference in the operation time for the two procedures, no major CBD injury and no mortality. The conversion rate at $13 \%$ was quite acceptable. 


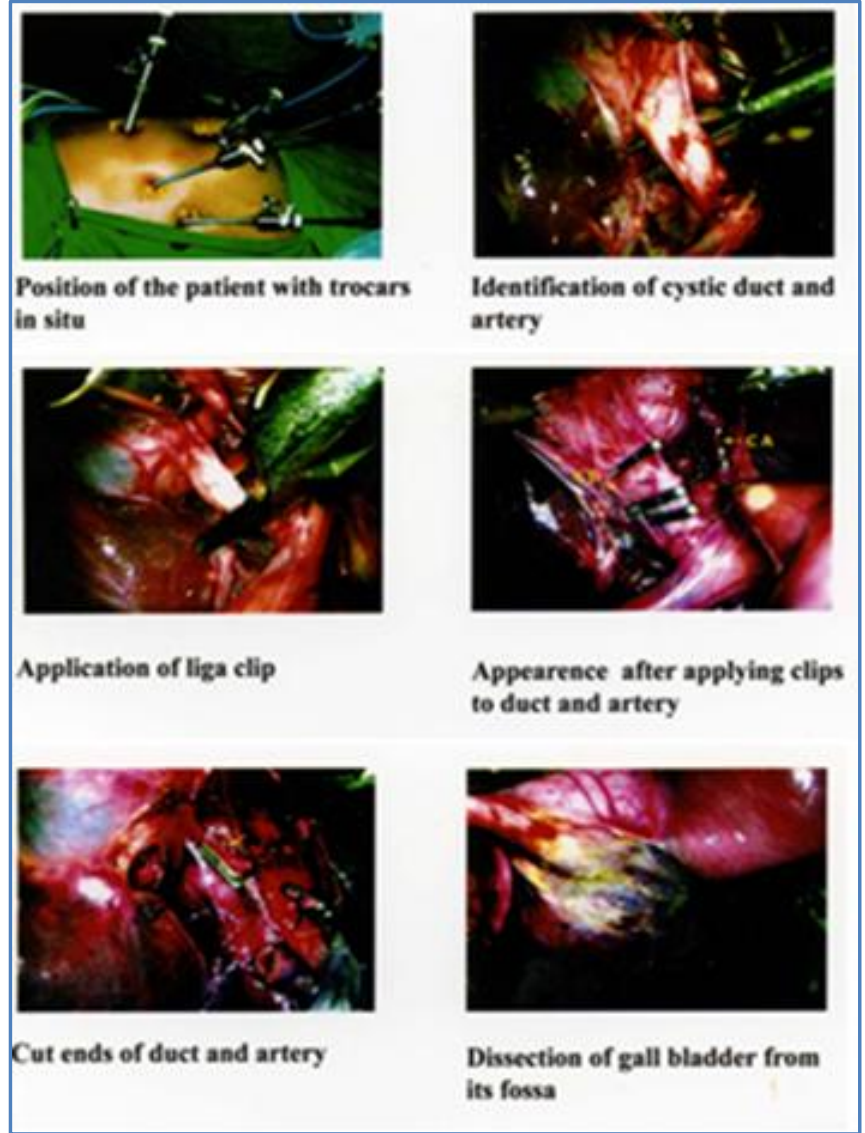

A comparative study of Laparoscopic \& Open Cholecystectomy

\section{BIBLIOGRAPHY:}

1. Bates, Merzer, Harrison. Symptomatic gallstone disease before and after cholecystectomy. Gut 1984; 24:A57980.

2. Langenbuch C. Ein Fall Von Extirpation der Gallenblase Wegen Choronischer Cholelithiasis, Heilung. Berl Klin Wochenschr 1882;19:725

3. National inpatient profile. Health care knowledge systems. Ann Arbor March 1989

4. Mcsherry. Cholecystectomy: the gold standard. Am J Surg 1989; 158:174-8

5. Garcia Valdez, Alminiva et al. Subcostal vs. midline incision in gallstone surgery: a prospective and randomized study. Br J Surg 1988; 75:473-75

6. Girard. Open cholecystectomy, morbidity and mortality as a reference standard. Can J Surg, Feb 1993; 36:75-80.

7. Cooper ED. Pathogenesis and treatment of gallstone disease. Gastro Clin N Am, March 1991.

8. Cgesyln-curtis, Russell R.C.G. New trends in gallstone management. Br J of Surg 1991; 98:14349.

9. Morgan Paul, Devlin et al. Length of stay for common surgical procedures. Br J of Surg 1987; Vol 74:884-9. 


\section{ORIGINAL ARTICLE}

10. Mouret. From the first laparoscopic cholecystectomy to the frontiers of laparoscopic surgery. Dig Surg 1991; 8:124-5.

11. NIH consensus development conference statement on gallstones and laparoscopic cholecystectomy. Am J Surg, April 1993; 165:390-96.

12. Jenkins PJ, Paterson HM, Parks RW.et al. Br J Surg.2007;94:1382-1385

\section{AUTHORS:}

1. Shekappa C. Malagimani

2. Somashekhar Samagandi

3. Ishwar Hosamani

4. Yashwanth C. T.

5. Vasanta S. C.

\section{PARTICULARS OF CONTRIBUTORS:}

1. Associate Professor, Department of Surgery, VIMS, Bellary.

2. Associate Professor, Department of Plastic Surgery, VIMS, Bellary.

3. Professor, Department of Surgery, KIMS, Hubli.

4. Post Graduate Student, Department of Surgery, VIMS, Bellary.
5. Assistant Professor, Department of Biostatistics, VIMS, Bellary.

\section{NAME ADDRESS EMAIL ID OF THE CORRESPONDING AUTHOR:}

Dr. Shekappa C. M, B/24, Staff Quarters, VIMS (OPD) Cantonment, Bellary.

E-mail:doc_shekar@yahoo.com

Date of Submission: 05/02/2014. Date of Peer Review: 06/02/2014. Date of Acceptance: 14/02/2014. Date of Publishing: 22/02/2014. 